\title{
Energy transport in Heisenberg chains beyond the Luttinger liquid paradigm
}

\author{
Andrea De Luca,, , 用Jacopo Viti, ${ }^{2}$, Leonardo Mazza, ${ }^{3, \text {, }}$ and Davide Rossini ${ }^{3}$, 8 \\ ${ }^{1}$ Laboratoire de Physique Théorique de l'ENS $\&$ Institut de Physique Theorique Philippe Meyer, Paris, France. \\ ${ }^{2}$ Laboratoire de Physique Théorique de l'ENS, CNRS \& Ecole Normale Supérieure de Paris, Paris, France. \\ ${ }^{3}$ NEST, Scuola Normale Superiore $\&$ Istituto Nanoscienze-CNR, Pisa, Italy.
}

(Dated: October 3, 2018)

\begin{abstract}
We study the energy transport between two interacting spin chains which are initially separated, held at different temperatures and subsequently put in contact. We consider the spin-1/2 XXZ model in the gapless regime and exploit its integrability properties to formulate an analytical Ansatz for the non-equilibrium steady state even at temperatures where the low-energy Luttinger liquid description is not accurate. We apply our method to compute the steady energy current and benchmark it both with the known low-energy limit and at higher temperatures with numerical simulations. We find an excellent agreement even at high temperatures, where the Luttinger liquid prediction is shown to fail.
\end{abstract}

PACS numbers: 05.30.-d, 05.50.+q, 74.40.Gh

Introduction. - The study of heat propagation is a fertile research field in condensed matter physics [1]. Up to few years ago, low-dimensional transport experiments have been always considered a prerogative of solid state nanowires. Any measurable current is modeled as a flow between incoherent and non-interacting reservoirs [2], so that transport phenomena in this scenario are well described within the Landauer-Büttiker approach [3]. However, the latest groundbreaking advances with cold atoms have challenged this paradigm: thermoelectric transport can now be studied with high degree of control and tunability in globally closed systems, where the interplay between interactions and coherence is potentially crucial [4].

A recent theoretical work [5] investigated two semiinfinite 1D reservoirs described by a conformal field theory $(\mathrm{CFT})$ and held at different temperatures [6]. At a certain time $t_{0}$, they are directly put in contact restoring translational invariance and evolved unitarily (see Fig. 1). There it is shown the existence of a non-equilibrium steady state (NESS) featuring an energy current that only depends on the central charge $c$ specifying the CFT of the reservoirs. CFT [7] describes the low-energy physics of 1D gapless systems and a standard framework to characterize electronic wires at low temperatures is the Luttinger Liquid (LL) 8], a CFT with $c=1$. It follows that the thermal current between 1D electron reservoirs has a universal low-temperature behavior. The presence of a persistent current and its universality have been rigorously proven in free models $[9,10$ and numerically verified in the scaling limit of critical spin chains [11].

However, the LL model describes the low-energy excitations of the interacting system [12, and its predictions are deemed to fail far from equilibrium and whenever too high temperatures are considered. Various numerical [13 18] and analytical studies [19 22] have attacked the problem, highlighting the peculiar effects of integrability. Moreover, recent experimental results [23 25] provided evidence of ballistic heat transport of quantum spin

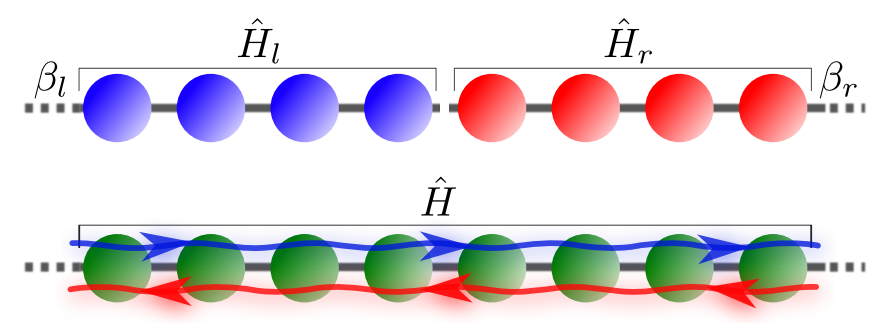

FIG. 1. Sketch of the non-equilibrium protocol: Two initially disconnected half chains of semi-infinite length, thermalized at different inverse temperatures $\beta_{l}$ and $\beta_{r}$, are connected at time $t_{0} \equiv 0$. A net energy current $\mathcal{J}$ flowing through the system is expected to appear once the steady state is established. At low temperature, this is interpreted as the effect of separate thermalization of the right/left moving excitations (lines with arrows).

excitations.

In this paper we study two reservoirs described by the XXZ spin-1/2 Hamiltonian in the gapless regime. This model is unitarily related to a system of interacting spinless fermions [12]. Its special integrability properties suggest the existence of a NESS energy current even at high temperatures [11, 26]. An analytical treatment based on the thermodynamic Bethe Ansatz has been put forward for the isotropic ferromagnet exploiting the low density of magnons at low-temperature [11. As expected, this approximation is not appropriate to reproduce the conformal regime considered here. We discuss an Ansatz for the NESS density matrix based on the quantumtransfer-matrix (QTM) formalism 27-29. We propose an analytical method to characterize the thermal current at arbitrary temperatures. In the low-temperature limit, our approach reproduces exactly the LL prediction [5]. Upon comparison with matrix-product-state (MPS) simulations [15, 30, the method is shown to be almost exact even at high temperature. In the latter regime, it discloses distinctive signatures of interactions in the energy 
transport of 1D fermionic systems.

The Model. - We consider the Hamiltonian

$$
\begin{aligned}
& \hat{H}=\hat{H}_{l}+\hat{H}_{r}+\hat{h}_{0}, \quad \hat{H}_{l}=\sum_{n<0} \hat{h}_{n}, \quad \hat{H}_{r}=\sum_{n>0} \hat{h}_{n}, \\
& \hat{h}_{n}=J\left[\hat{S}_{n}^{x} \hat{S}_{n+1}^{x}+\hat{S}_{n}^{y} \hat{S}_{n+1}^{y}+\Delta \hat{S}_{n}^{z} \hat{S}_{n+1}^{z}\right],
\end{aligned}
$$

$\hat{S}_{n}^{\alpha}$ being the $\alpha$-th component of the spin- $1 / 2$ on site $n\left(\hbar=k_{\mathrm{B}}=1\right)$; we focus on the critical phase $-1<$ $\Delta \leq 1$, parameterizing $\Delta=\cos \gamma, \gamma \in[0, \pi)$. Numerical results will be given for the antiferromagnetic regime $\gamma \in[0, \pi / 2]$. At the beginning, the system is separated into two independent halves held at different inverse temperatures $\beta_{r, l}$

$$
\rho_{0}=\mathcal{Z}^{-1} e^{-\beta_{l} \hat{H}_{l}} \otimes e^{-\beta_{r} \hat{H}_{r}}
$$

where $\mathcal{Z}$ ensures normalization of $\rho_{0}$. For times $t \geq 0$ the state is unitarily evolved with Hamiltonian $\hat{H}$ (1), so that the initially-separated reservoirs are put in contact and generate a heat flow (see Fig. 1).

The existence of a NESS requires the convergence of the long-time limit

$$
\lim _{t \rightarrow \infty} \lim _{N \rightarrow \infty} \operatorname{Tr}\left[\hat{\mathcal{O}} e^{-i \hat{H} t} \rho_{0} e^{i \hat{H} t}\right] \equiv\langle\hat{\mathcal{O}}\rangle_{\mathrm{NESS}}
$$

for any local observable $\hat{\mathcal{O}}$, where $N$ is the system size. Equation (33) formally defines a density matrix $\rho_{\mathrm{NESS}} \mathrm{em}-$ bodying all the local properties of the long-time dynamics.

In some peculiar situations the structure of $\rho_{\mathrm{NESS}}$ can be inferred from general symmetry arguments, as translational invariance. Suppose that the excitations of the system can be separated into two non-interacting sets with positive $(+)$ and negative $(-)$ momenta, so that $\hat{H}=\hat{H}_{+}+\hat{H}_{-}$and $\left[\hat{H}_{+}, \hat{H}_{-}\right]=0$ (here by positive we mean going from left to right, and vice-versa - see Fig. 1). Then $\rho_{\mathrm{NESS}}=\mathcal{Z}^{-1} e^{-\beta_{l} \hat{H}_{+}} \otimes e^{-\beta_{r} \hat{H}_{-}}$, i.e., the right (left) movers with positive (negative) momenta are separately thermalized at $\beta_{l}\left(\beta_{r}\right)$. This happens notably in any CFT, where the expectation value of the energy current operator in the middle of the chain $\hat{J}_{E}=(i / 2)\left[\hat{H}, \hat{H}_{r}-\hat{H}_{l}\right]$ is [5]

$$
\mathcal{J}=\left\langle\hat{J}_{E}\right\rangle_{\mathrm{NESS}}=\frac{\pi c}{12}\left(\beta_{l}^{-2}-\beta_{r}^{-2}\right),
$$

with $c=1$ for the critical XXZ model. However when the temperatures are increased, corrections appear due to irrelevant operators 31] that couple the right and left movers and spoil the pure conformal result (4).

The QTM approach. - We now explain the method to extend Eq. (4) to higher temperatures for the XXZ model. First we briefly recall how the QTM formalism allows to extract thermodynamical quantities at equilibrium, i.e. when $\beta_{l}=\beta_{r}=\beta$. Excitations of this spin chain can be interpreted as quasiparticles and quasiholes with energy 32 .

$$
\varepsilon(u)=\frac{\pi \Lambda}{2 \cosh (\pi u / 2)},
$$

where $\Lambda=2 J \sin \gamma / \gamma$ and $u \in(-\infty, \infty)$ parametrizes the first Brillouin zone, such that $u>0(u<0)$ corresponds to positive (negative) momenta. Thermodynamic quantities can be obtained knowing the quasiparticles and quasiholes occupation numbers $n(u)$ and $\bar{n}(u)$ at finite temperature. It is useful to rewrite $n \equiv b /(1+b)$ (and analogously for $\bar{n}$ ), where $b(u)$ and $\bar{b}(u)$ solve the nonlinear integral equation [28]

$$
\left[\begin{array}{l}
\log b \\
\log \bar{b}
\end{array}\right]=\left[\begin{array}{l}
s \\
s
\end{array}\right]+\left[\begin{array}{cc}
\mathcal{K} & -\mathcal{K}_{+} \\
-\mathcal{K}_{-} & \mathcal{K}
\end{array}\right] \star\left[\begin{array}{l}
\log (1+b) \\
\log (1+\bar{b})
\end{array}\right],
$$

where $f \star g$ is the usual convolution. At equilibrium the source therm $s(u)$ in (6) is $s(u)=-\beta \varepsilon(u)$. The kernel $\mathcal{K}(u-v)$ is the logarithmic derivative of the scattering phase between two quasi-particles or quasi-holes at different momenta. Explicitly one has

$$
\mathcal{K}(u)=\int_{-\infty}^{\infty} \frac{d w}{2 \pi} \frac{\sinh \left[\left(\frac{\pi}{\gamma}-2\right) w\right]}{2 \cosh w \sinh \left[\left(\frac{\pi}{\gamma}-1\right) w\right]} e^{i w u}
$$

and $\mathcal{K}_{ \pm}(u)=\mathcal{K}(u \pm 2 i)$. Notice that, for $\Delta=0(\gamma=\pi / 2)$, $\mathcal{K}$ vanishes and Eq. (6) reduces to the usual Fermi-Dirac distribution $n=1 /(1+\exp (\beta \varepsilon))$ for free fermions. The advantage of using the QTM approach and Eq. (6) lies in its low-temperature limit $\beta \rightarrow \infty$. As we will discuss below, at low temperatures Eq. (6) decouples into two independent equations determining the occupation numbers for excitations with positive $(u>0)$ and negative momenta $(u<0)$. In the $\Delta \rightarrow 0$ limit they correspond to the two chiral components of a free Dirac fermion.

In order to describe this out-of-equilibrium protocol, we propose to use Eq. (6) assuming a source term of the form $s(u)=-\beta(u) \varepsilon(u)$ with $\beta(u)=\beta_{r}\left(\beta_{l}\right)$ for $u<0$ $(u>0)$. Indeed, in this setting, the quasi-particles and quasi-holes can be supposed to be thermalized at large distances from the center of the system where they are infinitely far apart and interactions are negligible. Then, as it happens at equilibrium, bulk occupation numbers are modified by the phase shifts collected in each scattering process, which result in the convolution term of (6). Since this term is the effect of the microscopic processes appearing in an integrable model, it is natural to assume that it is not affected by the initial conditions. Despite this hypothesis, the proper derivation of the source term would require the exact solution of the dynamics at long times, an extremely challenging task even for integrable models. Our choice is exact at the free-fermion point $(\Delta=0)[10$ and, as we will see, yields the correct low-temperature behavior for all $\Delta$, which remained 


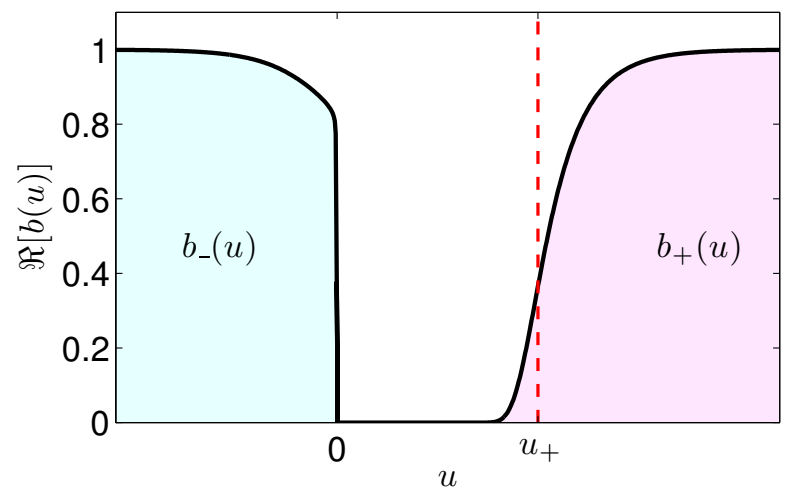

FIG. 2. The real part of $b(u)$ is shown vs $u$ for $\Delta=0.5$ and $\beta_{l}=10^{3}$, while $\beta_{r}=0$. The function is exponentially suppressed for $0<u \lesssim u_{+}$. The imaginary part similarly vanishes in the same domain.

elusive in the analytic approach of [1]. The idea of thermalizing independently right-left-moving quasi-particles was already stated in [5, 33.

The knowledge of $n(u)$ permits computing the expectation value in the NESS of any local operator [34, and in particular $\mathcal{J}$. A complete set of conserved quantities $\hat{\mathcal{Q}}_{m}\left(m \in \mathbb{N}^{+}\right)$in the XXZ chain can be defined iteratively 35 starting from the Hamiltonian $\hat{H} \equiv \hat{\mathcal{Q}}_{1}$ by repeated applications of the boost operator $\hat{\mathcal{B}}=\sum_{n} n \hat{h}_{n}$ : $\hat{\mathcal{Q}}_{m+1}=i\left[\hat{\mathcal{B}}, \hat{\mathcal{Q}}_{m}\right]$. Their expectation values in the NESS are extensive, i.e., $\left\langle\hat{\mathcal{Q}}_{m}\right\rangle \sim N q_{m}$. Because of translational invariance, the energy current $\mathcal{J}$ coincides with $-q_{2}$. Indeed, $\hat{J}_{E}=-\left(\hat{j}_{0}+\hat{j}_{-1}\right) / 2$, where $\hat{j}_{n}=i\left[\hat{h}_{n}, \hat{h}_{n-1}\right]$; by commuting $\hat{H}=\sum_{n} \hat{h}_{n}$ with $\hat{\mathcal{B}}$ one easily realizes that $\hat{\mathcal{Q}}_{2}=\sum_{n} \hat{j}_{n}$.

The explicit expression for $q_{m}$ is

$$
q_{m}=f_{m}+\int_{-\infty}^{\infty} d u \frac{n(u) a_{m}(u)+\bar{n}(u) \bar{a}_{m}(u)}{4 \cosh (\pi u / 2)},
$$

where the $f_{m}$ are constants vanishing for even $m$. Details on the derivation of (8) and on its relation with the generalized Gibbs ensemble [36, 37] are contained in the supplementary material. The auxiliary functions $a_{m}, \bar{a}_{m}$ solve the following linear integral equation

$$
\left[\begin{array}{l}
a_{m} \\
\bar{a}_{m}
\end{array}\right]=\left[\begin{array}{c}
\varepsilon_{m} \\
\varepsilon_{m}
\end{array}\right]+\left[\begin{array}{cc}
\mathcal{K} & -\mathcal{K}_{+} \\
-\mathcal{K}_{-} & \mathcal{K}
\end{array}\right] \star\left[\begin{array}{l}
n a_{m} \\
\bar{n} \bar{a}_{m}
\end{array}\right] .
$$

The function $\varepsilon_{m}(u)$ is the quasi-particle eigenvalue of the $m$-th conserved quantity, $\varepsilon_{m+1}(u)=\Lambda^{m} \varepsilon^{(m)}(u)$ where $\varepsilon^{(m)}$ is the $m$-th derivative of Eq. (5).

Eqs. (6) and (9) are suitable for analytical manipulations and can be easily solved numerically for $0 \leq \Delta \leq 1$ by iteration, treating convolutions in Fourier space. However, in the ferromagnetic regime, the numerical solution by iteration is problematic, as it happens already at equilibrium [26.

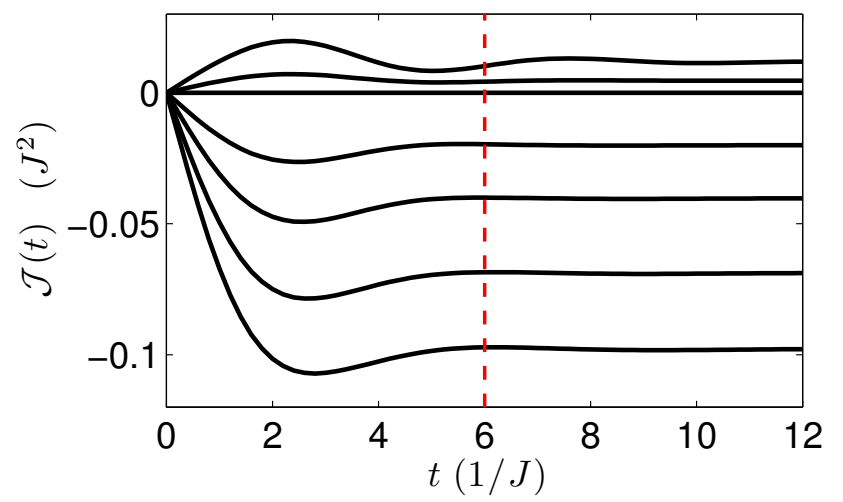

FIG. 3. Time evolution of the energy current $\mathcal{J}(t)$ for $\Delta=0.5$ and $\beta_{l}=4 J^{-1}$. Different lines refer to different values of $\beta_{r}$, from top to bottom: 8.0, 4.8, 4, 2.4, 1.6, 0.8, $0.04 J^{-1}$. Steadystate values are extrapolated by averaging over times $t \geq t_{\min }$ (see the vertical dashed line). Error bars in Fig. 4 indicate the range of values between the largest and the smallest computed value of $\mathcal{J}(t)$ for $t \geq t_{\min }$.

Low-temperature limit. - We first validate our Ansatz considering the limit $\beta_{l} \rightarrow \infty$ while $\beta_{r}$ remains finite. In this case, the function $b(u)$ is negligible in a finite range of values $u \in\left[0, u_{+}\right]$(see Fig. 2). The value $u_{+}=\frac{2}{\pi} \log \left[\pi \Lambda \beta_{l}\right]$ is estimated neglecting the second term in the r.h.s. of Eq. 6 and imposing $b\left(u_{+}\right) \sim 1$. It is natural to split $b(u)$ into two functions by defining $b_{+}(\xi) \equiv b\left(u_{+}+\frac{2}{\pi} \xi\right)$ and $b_{-}(\xi)=b\left(-\frac{2}{\pi} \xi\right)$ for positive $\xi$ and zero otherwise (respectively violet and cyan in Fig. 2). For the case of interest $\beta_{l} \rightarrow \infty\left(u_{+} \rightarrow \infty\right)$, Eq. (6) decouples into two separate equations for $b_{ \pm}$, because $\mathcal{K}(u)$ vanishes for large $u$. Moreover the equation for $b_{+}\left(b_{-}\right)$depends only on $\beta_{l}\left(\beta_{r}\right)$ and consequently the expectation value $q_{m}$ has the simple form

$$
q_{m}=f_{m}+q_{m}^{+}\left(\beta_{l}\right)+q_{m}^{-}\left(\beta_{r}\right),
$$

where $q_{m}^{ \pm}$can be computed as explained in the supplementary material. In particular, $q_{m}^{+}$is exactly obtained generalizing the so-called dilogarithm trick [26]

$$
q_{m}^{+}\left(\beta_{l}\right)=-\frac{\pi c_{m}}{12 \beta_{l}^{2}}
$$

with $c_{m+2}=(-\pi \Lambda / 2)^{m}$. Notice that $c_{2}=1$ and the contribution to the energy current $\mathcal{J}$ due to the right moving excitations is universal. Assuming a large $\beta_{r}$, one obtains a similar expression for $q_{m}^{-}$and the complete relation (4) is recovered.

An interesting outcome of this approach is the splitting of the chiral degrees of freedom whenever one of the two temperatures is small irrespectively of the other, as shown in Eq. (10). This explains why the factorization of the current approximately holds for all values of the temperatures, as numerically observed in [11. Intuitively, $u_{+}$can be considered as an effective Fermi point around which the relevant excitations are located. Due to the 

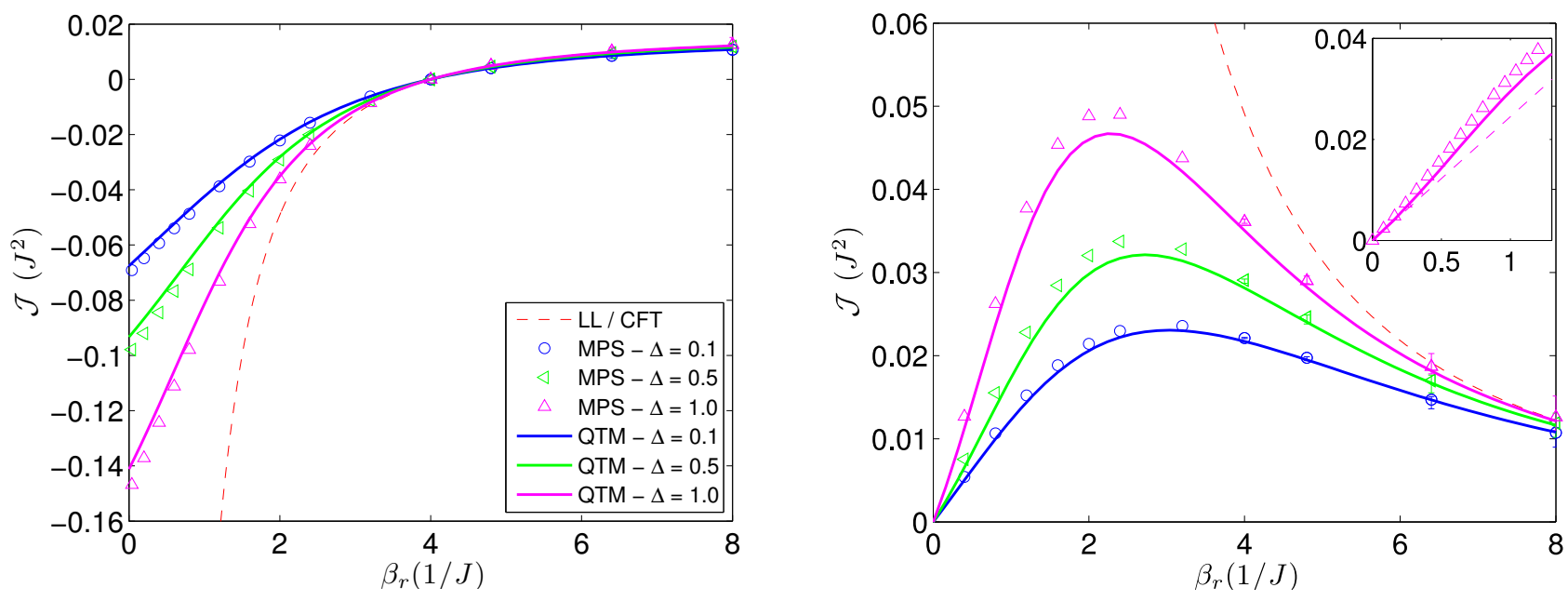

FIG. 4. Energy current $\mathcal{J}$ for several values of $\Delta$ as a function of $\beta_{r}$ for (a) $\beta_{l}=4 J^{-1}$ and (b) $\beta_{l}=\beta_{r} / 2$. The QTM results (continuous lines) are validated by the MPS data (symbols). When not shown, the numerical error bar is smaller than the marker (see Fig. 3). The CFT prediction (4) (dashed red line) describes the data only when both temperatures are small. The inset provides a zoom of the high-temperature. The dashed-line is the linear approximation of $\mathcal{J}$ for $\beta_{r}=0$ (see supplementary material). The QTM curve shows a change of concavity which also appears in the MPS data, thus proving that our Ansatz can capture even small details of the energy current.

locality of the interaction, excitations with a large momentum difference do not affect each other. Thus, even the left moving excitation closest in momentum to $u_{+}$ cannot be influenced by the right moving ones.

Higher temperatures. - To validate the predictions of our Ansatz at higher temperatures, we perform numerical simulations with an algorithm based on timedependent MPS [30. The initial thermal state $\rho_{0}$ is computed purifying the density matrix via the ancilla method [38]; the approach for real-time evolution of thermal states, introduced in Ref. [15, 39, is fundamental to reach sufficiently long times. We consider chains up to $N=100$ with open boundary conditions; finite-size effects are under control. The maximum allowed bond link is $D=3000$ and the truncation error per step is set to $10^{-10}$. The algorithm computes the real time evolution of the density matrix, i.e. $\rho(t) \equiv e^{-i \hat{H} t} \rho_{0} e^{i \hat{H} t}$.

In Fig. 3 we plot the energy current $\mathcal{J}(t) \equiv \operatorname{Tr}\left[\hat{J}_{E} \rho(t)\right]$ as a function of time. Upon waiting enough time, the system displays clear steady signatures. We interpret them as distinctive features of $\rho_{\mathrm{NESS}}$, even if the numerics cannot guarantee the formal existence of such limit: longer time scales are unaccessible, due to the exponentially increasing amount of needed resources. However we can estimate the steady-state limit by time-averaging $\mathcal{J}(t)$ from a given $t_{\min }$ up to the longest reached time. Figure 4 compares these values with those derived from the analytical Ansatz. The agreement is excellent even in the high-temperature region, where the LL prediction (4) completely fails, as the latter requires both $\beta_{l}$ and $\beta_{r}$ to be large. The QTM method is less accurate far from equilibrium when the temperatures are of order $J$ (see the right panel for $\beta_{l}=\beta_{r} / 2 \sim 2 J^{-1}$ ), though here the relative inaccuracy is always found to be less than $10 \%$. It is difficult to address the steady state for lowtemperatures with MPS because of inaccessible long equilibration times, thus yielding non-negligible error bars on the estimated value of $\mathcal{J}$. However, in this regime the QTM method is guaranteed to work by the presented analytical considerations.

The data confirm the intuitive expectation that the current is larger in situations where $\beta_{l}$ strongly differs from $\beta_{r}$. The non-monotonous behavior of $\mathcal{J}\left(\beta_{r}\right)$ in the right panel of the figure follows from the competition between this tendency and the fact that for $\beta_{r}=0$ the system is at equilibrium. Interestingly, in both panels we observe that strong interactions (large values of $|\Delta|$ ) enhance the current.

Concluding remarks. - We developed an analytic QTM formalism that is able to describe the steady-state energy current flowing between two interacting XXZ chains integrable through Bethe Ansatz. The method is predictive even far from equilibrium and at high temperatures, where the Landauer-Büttiker approach cannot be employed.

This non-equilibrium protocol can be realized in forthcoming cold-atom experiments [4] where the distinctive features of the energy current predicted by our technique could be effectively measured. The NESS is observable on a time scale of order $L / v$, where $L$ is the system length and $v$ the typical quasi-particle velocity; for larger times, the system will equilibrate and the current will vanish 40 .

Extensions to other experimentally realizable situations, i.e. the unbalance of chemical potentials, are possible within the same formalism. Moreover, the problem 
of transport in the Lieb-Liniger model is under consideration by the authors.

We believe that the peculiar properties of cold atomic systems will soon create new out-of-equilibrium paradigms, where interactions and unitary dynamics are relevant; these studies will pave the way for their understanding, well beyond the commonly employed linearresponse approach [42].

Acknowledgements. - We are indebted to R. Fazio for enlightening comments and support. We also acknowledge fruitful discussions with D. Bernard, P. Calabrese, B. Doyon, F. Essler, J. Moore and in particular A. Klümper. This work was supported by Italian MIUR via FIRB Project RBFR12NLNA, and by Regione Toscana POR FSE 2007-2013. A.D.L. thanks Scuola Normale Superiore for hospitality.

* andrea.deluca@lpt.ens.fr

† jacopo.viti@lpt.ens.fr

‡ leonardo.mazza@sns.it

$\S$ davide.rossini@sns.it

[1] Y. M. Blanter and M. Büttiker, Phys. Reports 336, 1 (2000).

[2] C. L. Kane and M. P. A. Fisher, Phys. Rev. Lett. 76, 3192 (1996); R. Fazio, F. W. J. Hekking, and D. E. Khmelnitskii, ibid. 80, 5611 (1998).

[3] S. Datta, Electronic transport in mesoscopic systems (Cambridge university press, 1997).

[4] M. Gring, M. Kuhnert, T. Langen, T. Kitagawa, B. Rauer, M. Schreitl, I. Mazets, D. A. Smith, E. Demler, and J. Schmiedmayer, Science 337, 1318 (2012) J.-P. Brantut, J. Meineke, D. Stadler, S. Krinner, and T. Esslinger, Science 337, 1069 (2012); S. Krinner, D. Stadler, D. Husmann, J.-P. Brantut, and T. Esslinger, (2014), arXiv:1404.6400

[5] D. Bernard and B. Doyon, J. Phys. A 45, 362001 (2012); in Annales Henri Poincaré (Springer, 2013) pp. 1-49.

[6] H. Spohn and J. L. Lebowitz, Commun. Math. Phys. 54, 97 (1977).

[7] P. Di Francesco, P. Mathieu, and D. Senechal, Conformal field theory (Springer, 1997).

[8] F. Haldane, J. Phys. C: Solid State 14, 2585 (1981); Phys. Rev. Lett. 47, 1840 (1981).

[9] T. Ho and H. Araki, Proc. Steklov Inst. Math. 228, 203 (2000); S. Tasaki, Chaos, Solitons \& Fractals 12, 2657 (2001); W. H. Aschbacher and C.-A. Pillet, J. Stat. Phys. 112, 1153 (2003).

[10] A. De Luca, J. Viti, D. Bernard, and B. Doyon, Phys. Rev. B 88, 134301 (2013).

[11] C. Karrasch, R. Ilan, and J. E. Moore, Phys. Rev. B 88, 195129 (2013).

[12] T. Giamarchi, Quantum physics in one dimension (Clarendon Press, 2004).

[13] F. Heidrich-Meisner, A. Honecker, D. C. Cabra, and W. Brenig, Phys. Rev. B 68, 134436 (2003).

[14] F. Heidrich-Meisner, A. Honecker, and W. Brenig, Eur. Phys. J. ST 151, 135 (2007).
[15] C. Karrasch, J. H. Bardarson, and J. E. Moore, Phys. Rev. Lett. 108, 227206 (2012); New J. Phys. 15, 083031 (2013).

[16] C. Karrasch, J. Hauschild, S. Langer, and F. HeidrichMeisner, Phys. Rev. B 87, 245128 (2013).

[17] C. Karrasch, J. Moore, and F. Heidrich-Meisner, Phys. Rev. B 89, 075139 (2014).

[18] L. Bonnes, F. H. Essler, and A. M. Läuchli, (2014), arXiv:1404.4062

[19] X. Zotos, F. Naef, and P. Prelovsek, Phys. Rev. B 55, 11029 (1997).

[20] P. Jung, R. W. Helmes, and A. Rosch, Phys. Rev. Lett. 96, 067202 (2006).

[21] J. Sirker, R. G. Pereira, and I. Affleck, Phys. Rev. Lett. 103, 216602 (2009); Phys. Rev. B 83, 035115 (2011).

[22] T. Prosen, Phys. Rev. Lett. 106, 217206 (2011); T. Prosen and E. Ilievski, 111, 057203 (2013); T. Prosen, Phys. Rev. E 89, 012142 (2014).

[23] A. Sologubenko, T. Lorenz, H. Ott, and A. Freimuth, J. Low Temp. Phys. 147, 387 (2007).

[24] C. Hess, Eur. Phys. J. ST 151, 73 (2007).

[25] N. Hlubek, P. Ribeiro, R. Saint-Martin, A. Revcolevschi, G. Roth, G. Behr, B. Büchner, and C. Hess, Phys. Rev. B 81, 020405 (2010).

[26] A. Klümper and K. Sakai, Journal of Physics A: Mathematical and General 35, 2173 (2002).

[27] A. Klümper, Z. Phys. B Condens. Matter 91, 507 (1993).

[28] A. Klümper and D. C. Johnston, Phys. Rev. Lett. 84, 4701 (2000).

[29] A. Klümper, in Quantum Magnetism, Lecture Notes in Physics (2004) p. 349.

[30] U. Schollwöck, Ann. Phys. 326, 96 (2011).

[31] S. Lukyanov, Nuclear Physics B 522, 533 (1998).

[32] L. Samaj and Z. Bajnok, Introduction to the Statistical Physics of Integrable Many-body Systems (Cambridge University Press, 2013).

[33] B. Doyon, (2012), arXiv:1212.1077; O. Castro-Alvaredo, Y. Chen, B. Doyon, and M. Hoogeveen, J. Stat. Mech. Theor. Exp. 2014, P03011 (2014).

[34] F. Göhmann, A. Klümper, and A. Seel, Journal of Physics A: Mathematical and General 37, 7625 (2004).

[35] M. Grabowski and P. Mathieu, Annals of Physics 243, 299 (1995).

[36] B. Pozsgay, Journal of Statistical Mechanics: Theory and Experiment 2013, P07003 (2013).

[37] M. Fagotti and F. H. Essler, J. Stat. Mech. Theor. Exp. 2013, P07012 (2013).

[38] A. E. Feiguin and S. R. White, Phys. Rev. B 72, 220401(R) (2005).

[39] D. Kennes and C. Karrasch, (2014), arXiv:1404.3704.

[40] M. Collura and D. Karevski, Phys. Rev. B 89, 214308 (2014)

[41] A. Klümper, Annalen der Physik 504, 540 (1992) .

[42] Ch. Greiner, C. Kollath, and A. Georges, arXiv:1209.3942, arXiv:1406.4632; H. Kim and D. A. Huse, Phys. Rev. A 86, 053607 (2012); E. L. Hazlett, L.-C. Ha, and C. Chin, arXiv:1306.4018; A. Rancon, C. Chin, and K. Levin, arXiv:1311.0769; G. Benenti, G. Casati, T. Prosen, and K. Saito, arXiv:1311.4430 (2013); C.-C. Chien, M. Di Ventra and M. Zwolak, arXiv:1403.0511 (2014). 


\section{Supplementary Material for EPAPS \\ Energy transport in Heisenberg chains beyond the Luttinger liquid paradigm}

\section{QUANTUM TRANSFER MATRIX AND GENERALIZED GIBBS ENSEMBLE}

Here, we discuss how our Ansatz for the stationary state out-of-equilibrium can be interpreted as GGE. Let us consider the following non-normalized density matrix

$$
\rho=e^{-\sum_{m=1}^{\infty} \lambda_{m} \hat{\mathcal{Q}}_{m}},
$$

where $\hat{\mathcal{Q}}_{m}$ are mutually commuting operators defined in the text by means of the boost operator and the parameters $\lambda_{m} \in \mathbb{R}$ are suitably chosen Lagrange multipliers. Given the quantum state (S1), the QTM formalism introduced in 27, 41] and recently considered in [36, 37, allows computing the generalized free-energy

$$
\phi_{\rho}(\{\lambda\}) \equiv \log \operatorname{Tr}\left[e^{-\sum_{m=1}^{\infty} \lambda_{m} \hat{\mathcal{Q}}_{m}}\right] .
$$

In the particle-hole setup [27, one has explicitly

$$
\phi_{\rho}(\{\lambda\})=-\sum_{m=1}^{\infty} \lambda_{m} f_{m}+\int_{-\infty}^{\infty} d u \frac{\log [B(u) \bar{B}(u)]}{4 \cosh (\pi u / 2)},
$$

where $B=1+b$ and $\bar{B}=1+\bar{b}$. The functions $b, \bar{b}$ are the solutions of the non-linear integral equation (6) setting

$$
s(u)=-\sum_{m=1}^{\infty} \lambda_{m} \varepsilon_{m}(u)
$$

and the constants $f_{m}$ are the expectation values of the charges $\mathcal{Q}_{m}$ in the ground-state, given by

$$
f_{m}=\pi \gamma \Lambda^{m} \int_{-\infty}^{\infty} d \omega(i \omega)^{m-1}\left[\frac{\tanh \gamma \omega}{\tanh \pi \omega}-1\right]
$$

The QTM formalism can be used to obtain expectation values for all the conserved charges inside the state (S1). For instance for $\hat{\mathcal{Q}}_{m}$, we modify the free-energy as

$$
\phi_{\rho}(\{\lambda\}, \mu) \equiv \log \operatorname{Tr}\left[\rho e^{\mu \hat{\mathcal{Q}}_{m}}\right]
$$

and it can be obtained shifting the $k$-th Lagrange multiplier $\lambda_{m} \rightarrow \lambda_{m}-\mu$. Finally the expectation value of the charge density $q_{m}$ is given by the derivative

$$
q_{m}=\left.\partial_{\mu} \phi_{\rho}(\{\lambda\}, \mu)\right|_{\mu=0} .
$$

In principle, this value can be obtained computing (S6) numerically solving (6) for different $\mu$ and then using (S7). However, a numerically more stable procedure is to explicitly differentiate $\phi_{\rho}(\{\lambda\}, \mu)$ with respect to $\mu$ directly from its expression $(\mathrm{S} 3$. One obtains 8 where

$$
a_{m}=\left.\partial_{\mu} \log b(u)\right|_{\mu=0} .
$$

This last quantity solves (9), which is derived differentiating (6) with respect to $\mu$ at $\mu=0$.

In our approach the Lagrange multipliers in (S1) are implicitly fixed by the source term, that we choose to be

$$
s(u)=-\left[\beta_{l} \theta(u)+\beta_{r} \theta(-u)\right] \varepsilon(u) .
$$

To show that this can be written in the form (S1), we look for a set of $\lambda$ 's solving the equation

$$
\sum_{m=1}^{\infty} \lambda_{m} \varepsilon_{m}(u)=-s(u) \text {. }
$$


Denoting $\tilde{f}(q)$ the Fourier transform of $f(u)$

$$
f(u)=\int_{-\infty}^{\infty} \frac{d q}{2 \pi} e^{i q x} \tilde{f}(q),
$$

and using $\varepsilon_{m+1}(u)=\Lambda^{m} \varepsilon^{(m)}(u)$, we have

$$
\tilde{\varepsilon}_{m}(q)=(i q \Lambda)^{m-1} \tilde{\varepsilon}(q)
$$

and $(\mathrm{S} 10)$ is then solved by

$$
\lambda_{m+1}=-\left.\left(\frac{1}{i \Lambda}\right)^{m} \frac{1}{m !} \frac{d^{m}}{d q^{m}} \frac{\tilde{s}(q)}{\tilde{\varepsilon}(q)}\right|_{q=0} .
$$

Notice that all the derivatives are well defined since $\int_{-\infty}^{\infty} d u u^{k} \varepsilon(u)<\infty$ and the parameters $\lambda_{m}$ are fixed uniquely. Convergence of the LHS of $(\mathrm{S} 10)$ is pointwise with the exception of the point $u=0$.

\section{CONFORMAL LIMIT}

In this Appendix we derive the results 1011 . Let us consider the functions $b_{+}(\xi)=b\left(u_{+}+\frac{2}{\pi} \xi\right)$ and $b_{-}(\xi)=b\left(-\frac{2}{\pi} \xi\right)$ for positive $\xi$ and zero otherwise. The same definitions are used for the bar-quantities. For large $\beta_{l}, s\left(u_{+}+\frac{2}{\pi} \xi\right) \simeq-e^{-\xi}$ and (6) can be reduced to

$$
\begin{aligned}
& {\left[\begin{array}{l}
\log b_{+}(\xi) \\
\log \bar{b}_{+}(\xi)
\end{array}\right]=-\left[\begin{array}{l}
e^{-\xi} \\
e^{-\xi}
\end{array}\right]+\left[\begin{array}{cc}
\tilde{\mathcal{K}} & -\tilde{\mathcal{K}}_{\mp} \\
-\tilde{\mathcal{K}}_{ \pm} & \tilde{\mathcal{K}}
\end{array}\right] \star\left[\begin{array}{l}
\log B_{+}(\xi) \\
\log \bar{B}_{+}(\xi)
\end{array}\right],} \\
& {\left[\begin{array}{l}
\log b_{-}(\xi) \\
\log \bar{b}_{-}(\xi)
\end{array}\right]=\beta_{r}\left[\begin{array}{l}
\varepsilon\left(\frac{2 \xi}{\pi}\right) \\
\varepsilon\left(\frac{2 \xi}{\pi}\right)
\end{array}\right]+\left[\begin{array}{cc}
\tilde{\mathcal{K}} & -\tilde{\mathcal{K}}_{+} \\
-\tilde{\mathcal{K}} & \tilde{\mathcal{K}}
\end{array}\right] \star\left[\begin{array}{l}
\log B_{-} \\
\log \bar{B}_{-}
\end{array}\right],}
\end{aligned}
$$

where $B_{ \pm}(\xi)=1+b_{ \pm}(\xi)$ and the kernels $\tilde{\mathcal{K}}(\xi)=\frac{2}{\pi} \mathcal{K}\left(\frac{2 \xi}{\pi}\right)$ and $\tilde{\mathcal{K}}_{ \pm}(\xi)=\frac{2}{\pi} \mathcal{K}\left(\frac{2 \xi}{\pi} \pm 2 i\right)$. Notice that $\tilde{\mathcal{K}}(\xi)=\tilde{\mathcal{K}}(-\xi)$. Moreover we set $a_{m}^{+}(\xi)=a_{m}\left(u_{+}+\frac{2}{\pi} \xi\right)$ and $a_{m}^{-}(\xi)=a_{m}\left(-\frac{2}{\pi} \xi\right)$. Eq. 99 simplifies for large $\beta_{l}$ to

$$
\begin{aligned}
& {\left[\begin{array}{l}
a_{m}^{+}(\xi) \\
\bar{a}_{m}^{+}(\xi)
\end{array}\right]=\frac{1}{\beta_{l}}\left(-\frac{\pi \Lambda}{2}\right)^{m-1}\left[\begin{array}{c}
e^{-\xi} \\
e^{-\xi}
\end{array}\right]+\left[\begin{array}{cc}
\tilde{\mathcal{K}} & -\tilde{\mathcal{K}}_{\mp} \\
-\tilde{\mathcal{K}}_{ \pm} & \tilde{\mathcal{K}}
\end{array}\right] \star\left[\begin{array}{l}
n_{+} a_{m}^{+}(\xi) \\
\bar{n}_{+} \bar{a}_{m}^{+}(\xi)
\end{array}\right],} \\
& {\left[\begin{array}{l}
a_{m}^{-}(\xi) \\
\bar{a}_{m}^{-}(\xi)
\end{array}\right]=\left[\begin{array}{c}
\varepsilon_{m}\left(-\frac{2 \xi}{\pi}\right) \\
\varepsilon_{m}\left(\frac{2 \xi}{\pi}\right)
\end{array}\right]+\left[\begin{array}{cc}
\tilde{\mathcal{K}}^{(} & -\tilde{\mathcal{K}}_{\mp} \\
-\tilde{\mathcal{K}}_{ \pm} & \tilde{\mathcal{K}}^{\mp}
\end{array}\right] \star\left[\begin{array}{c}
n_{-} a_{m}^{-}(\xi) \\
\bar{n}_{-} \bar{a}_{m}^{-}(\xi)
\end{array}\right] .}
\end{aligned}
$$

where $n_{ \pm}=b_{ \pm} /\left(1+b_{ \pm}\right)$. Combining $(\mathrm{S} 14$, S16), one realizes that

$$
a_{m}^{+}(\xi)=\frac{1}{\beta_{l}}\left(-\frac{\pi \Lambda}{2}\right)^{m-1} \frac{d}{d \xi} \log b_{+}(\xi)
$$

Rewriting (8) with the functions $n_{ \pm}$and $a_{ \pm}$, we deduce the decomposition

$$
q_{m}=f_{m}+\underbrace{\frac{2}{\pi} \int_{0}^{\infty} \frac{n_{-}(\xi) a_{m}^{-}(\xi)}{4 \cosh \xi} d \xi}_{q_{m}^{-}\left(\beta_{r}\right)}+\underbrace{\frac{1}{\pi^{2} \Lambda \beta_{l}} \int_{-\infty}^{\infty} e^{-\xi} n_{+}(\xi) a_{m}^{+}(\xi) d \xi}_{q_{m}^{+}\left(\beta_{l}\right)}+\ldots
$$

where for simplicity we omit the bar-terms that have a similar decomposition, thus recovering the factorized form (10). The expression for $q_{m}^{+}$in 10 ) in the large- $\beta_{l}$ limit can be integrated by parts with the aid of (S18) to get

$$
q_{m}^{+}\left(\beta_{l}\right) \sim-\frac{c_{m}}{2 \pi \beta_{l}^{2}} \int_{\mathbb{R}} d \xi e^{-\xi} \log \left[B_{+}(\xi) \bar{B}_{+}(\xi)\right],
$$

where $c_{m+2}=(-\pi \Lambda / 2)^{m}$. The integral in $(\mathrm{S} 20)$ can be computed exactly [26]. Indeed, taking the $\xi$-derivative in (S14), multiplying the result on the left by $\left[\log B_{+}, \log \bar{B}_{+}\right]$and finally integrating one obtains

$$
\int_{\mathbb{R}} d \xi e^{-\xi} \log \left[B_{+}(\xi) \bar{B}_{+}(\xi)\right]=2 \int_{b_{+}(-\infty)}^{b_{+}(\infty)} \frac{d b}{b} \log (1+b) .
$$

Observing that $b_{+}(-\infty)=0$ and $b_{+}(\infty)=1$ and recalling $\int_{0}^{1} d u \log (1+u) u^{-1}=\pi^{2} / 12$, the result 11 now follows. 


\section{HIGH TEMPERATURE LIMIT}

We parameterize $\beta_{r}=\eta \beta_{l}=\beta$ and consider the limit $\beta \rightarrow 0$ where $b=1+\left.\beta \partial_{\beta} b\right|_{\beta=0}+o(\beta)$ and similarly for $\bar{b}$; then linearizing (6) we obtain the following integral equation for the auxiliary function derivatives at $\beta=0$

$$
\left[\begin{array}{l}
\left.\partial_{\beta} b\right|_{\beta=0} \\
\left.\partial_{\beta} \bar{b}\right|_{\beta=0}
\end{array}\right]=-\frac{1}{2}\left[\begin{array}{l}
\varepsilon(u)[\eta+(1-\eta) \theta(-u)] \\
\varepsilon(u)[\eta+(1-\eta) \theta(-u)]
\end{array}\right]+\frac{1}{2}\left[\begin{array}{cc}
\mathcal{K} & -\mathcal{K}_{+} \\
-\mathcal{K}_{-} & \mathcal{K}
\end{array}\right] \star\left[\begin{array}{l}
\left.\partial_{\beta} b\right|_{\beta=0} \\
\left.\partial_{\beta} \bar{b}\right|_{\beta=0}
\end{array}\right] .
$$

The current slope at high temperatures can be determined from (8) expanding the integrand at order $\beta$; one has $\mathcal{J}(\beta)=\beta \kappa+o(\beta)$ with

$$
\kappa=\int_{-\infty}^{\infty} \frac{d u}{16 \cosh (\pi u / 2)}\left[\left.\partial_{\beta} b\right|_{\beta=0} a_{0}+\left.2 \partial_{\beta} a\right|_{\beta=0}+\left.\partial_{\beta} \bar{b}\right|_{\beta=0} \bar{a}_{0}+\left.2 \partial_{\beta} \bar{a}\right|_{\beta=0}\right]
$$

where $a(\beta)=a_{0}+\left.\beta \partial_{\beta} a\right|_{\beta=0}+o(\beta)$ and analogously for $\bar{a}$. Linear integral equations for $a_{0}, \bar{a}_{0}$ and $\left.\partial_{\beta} a\right|_{\beta=0},\left.\partial_{\beta} \bar{a}\right|_{\beta=0}$ are derived from $(9)$, they read

$$
\begin{aligned}
& {\left[\begin{array}{l}
a_{0} \\
\bar{a}_{0}
\end{array}\right]=\left[\begin{array}{l}
\varepsilon_{2} \\
\varepsilon_{2}
\end{array}\right]+\frac{1}{2}\left[\begin{array}{cc}
\mathcal{K} & -\mathcal{K}_{+} \\
-\mathcal{K}_{-} & \mathcal{K}
\end{array}\right] \star\left[\begin{array}{l}
a_{0} \\
\bar{a}_{0}
\end{array}\right],} \\
& {\left[\begin{array}{l}
\left.\partial_{\beta} a\right|_{\beta=0} \\
\left.\partial_{\beta} \bar{a}\right|_{\beta=0}
\end{array}\right]=\frac{1}{4}\left[\begin{array}{cc}
\mathcal{K} & -\mathcal{K}_{+} \\
-\mathcal{K}_{-} & \mathcal{K}
\end{array}\right] \star\left[\begin{array}{l}
\left.\partial_{\beta} b\right|_{\beta=0} a_{0}+\left.2 \partial_{\beta} a\right|_{\beta=0} \\
\left.\partial_{\beta} \bar{b}\right|_{\beta=0} \bar{a}_{0}+\left.2 \partial_{\beta} \bar{a}\right|_{\beta=0}
\end{array}\right] .}
\end{aligned}
$$

Eq. S24 can be solved analytically [26, whereas 222 and S25 only numerically. We finally set $\eta=1 / 2$ and employed (S23) to reproduce the linear behavior of the current plotted in the inset of Fig. 4 . 